\title{
Vers une typologie des dispositifs hybrides de formation en enseignement supérieur
}

Une typologie des dispositifs hybrides

\author{
R. Burton1 - S. Borruat2 - B. Charlier2 - N. Coltice3 \\ N. Deschryver4 - F. Docq5 - J. Eneau 7 - G. Gueudet7 \\ G. Lameul6 - M. Lebrun5 - A. Lietart5 - M. Nagels7 \\ D. Peraya4 - A. Rossier2 - E. Renneboog1 - E. Villiot-Leclercq7
}

RÉSUMÉ. L'évaluation des effets des dispositifs hybrides de formation en enseignement supérieur nécessite un cadre descriptif pour en comprendre les effets différenciés. Dans cette perspective, le présent article propose d'établir une typologie des dispositifs hybrides de formation à partir de leurs caractéristiques pédagogiques, organisationnelles et matérielles (modalités d'articulation des phases présentielles et distantes, accompagnement humain, formes de médiatisation et médiation liées à l'utilisation d'un environnement technopédagogique, degré d'ouverture). Il rend également compte d'un point de vue méthodologique, de la manière dont les chercheurs investis dans le projet européen HySup (2009-12) ont progressivement élaboré ce cadre et en ont fait une première expérimentation.

ABSTRACT. Assessing the effects of hybrid system requires a descriptive framework to understand and differentiate their effects. In this perspective, this article proposes a typology of hybrid system from their educational, organizational and material characteristics (modalities of articulation of presential and distant phases, teaching approaches, human support, forms of media and mediation related to the use of a techno-environment, degree of openness).

MOTS-CLÉS : typologie, dispositif hybride de formation, analyse de clusters, pédagogie universitaire. KEYWORDS:

typology, hybrid learning system, cluster analysis, higher education.

Paru dans: Distances et savoirs. Volume 9 - n 1/2011, pages 69 à 96 http://www.cned.fr/ds/volume-9/numero-1/

1. EMACS, Université du Luxembourg

Route de Diekirch L-7220 Walferdange, Luxembourg

\{reginald.burton; ellen.renneboog\}@uni.lu

2. Université de Fribourg

Boulevard de Pérolles 901700 Fribourg, Suisse

\{bernadette.charlier; stéphanie.borruat; annick.rossier\}@unifr.ch

3. Université Claude Bernard Lyon 1

43, Boulevard du 11 Novembre 191869622 Villeurbanne cedex, France

nicolas.coltice@recherche.univ-lyon1.fr

4. TECFA, Université de Genève

40 Boulevard du Pont d'Arve 1205 Genève 4, Suisse

deschryver.nathalie@gmail.com; daniel.peraya@unige.ch

5. Université Catholique de Louvain

54, UCL-IPM Grand Rue B-1348 Louvain-La-Neuve, Belgique

\{francoise.docq; marcel.lebrun\}@uclouvain.be ; alietart@gmail.com

6. Université Rennes 2

54310, Rue Saint Malo Cs 35043 F-Rennes cedex, France

genevieve.lameul@uhb.fr; marc.nagels@free.fr;ghislaine.gueudet@bretagne.iufm.fr

7. Université Joseph Fourier

30, avenue Marcelin Berthelot 38100 Grenoble, France emmanuelle.villiot-leclercq@ujf-

grenoble.fr 


\section{Avant-propos}

Cette contribution est le produit - encore partiel, d'une recherche collaborative menée dans le cadre d'un projet européen par six groupes de chercheurs francophones. Au-delà de l'intérêt de la subvention européenne, il résulte de la volonté de ses initiateurs (B. Charlier, N. Coltice, N. Deschryver et D. Peraya) de comprendre les effets des dispositifs auxquels ils contribuent depuis de nombreuses années comme concepteurs ou formateurs. En effet, ce sont nos observations empiriques d'effets récurrents de mêmes types de dispositifs appliqués dans des contextes universitaires différents - la Suisse et la Belgique, par exemple - qui ont suscité notre volonté d'essayer de comprendre leurs effets en fonction de leurs caractéristiques propres. Pour cela, il s'agissait de changer d'échelle et de s'associer à d'autres partenaires pour mener tout d'abord une recherche permettant de décrire des types ou des configurations de dispositifs appréhendés dans toute leur complexité, et dans un second temps, de chercher à en identifier les effets sur la qualité de l'apprentissage des étudiants, le développement professionnel des enseignants et le changement institutionnel. Ainsi cet article présente la première partie de cette recherche pouvant être qualifiée pour son ensemble de «mixed-methods » (Leech, Dellinger, Brannagan et Tanaka, 2010), ses méthodes, ses instruments de recueil de données et ses premiers résultats.

\section{Problématique et état de la recherche}

Depuis une dizaine d'années, l'usage des plateformes d'enseignement à distance se répand largement dans les universités européennes. Aussi assistons-nous à une transformation des dispositifs de formation traditionnels vers de nouveaux dispositifs « hybrides » (Charlier, Deschryver et Peraya, 2006). Par dispositif « hybride», nous entendions tout dispositif de formation qui se caractérise par la présence de dimensions innovantes (accompagnement humain, modalités d'articulation présence - distance...) liées à la mise à distance. Le dispositif hybride, parce qu'il suppose l'utilisation d'un environnement technopédagogique, repose sur des formes complexes de médiatisation et de médiation.

Mis à part des enquêtes de satisfaction menées auprès des étudiants, il y a aujourd'hui peu de données témoignant d'un effet positif de ces transformations sur la qualité des processus d'enseignement/apprentissage. Les métarecherches pionnières dans le domaine de l'enseignement assisté par ordinateur de (Kulik et Cohen, 1980) ont montré l'importance des dimensions non technologiques dans l'analyse des effets : les légères différences observées sont bien souvent entachées d'une variance importante ou alors noyées dans un bruit de fond lié à la variété des disciplines, aux différentes méthodes encadrant les outils ainsi qu'aux modalités d'évaluations des apprentissages réalisés (1980). C'est assez récemment que l'attention a été attirée sur la nécessité de tenir compte du dispositif pédagogique encadrant les outils et de l'attention pédagogique rendue encore plus indispensable dans les dispositifs qui s'appuient sur les technologies : une pédagogie rigoureuse est une condition incontournable pour que les TIC (technologies de l'information et de la communication) tiennent leurs promesses (Clark, 1994 ; Tardif, 1996). C'est ainsi que l'alignement pédagogique (Biggs, 1996) des objectifs, des méthodes et des évaluations que nous complétons par les outils technologiques utilisés (Lebrun, 2007) est présenté comme une condition d'un impact positif des TIC : « Our review provides convincing evidence that information technology can enhance learning when the pedagogy is sound, and where there is a good match of technology, techniques and objectives » (Kadiyala et Crynes, 2000). Cela nous paraît essentiel pour éviter la part aléatoire de ces impacts que décrivait Morgan (2003) : «There is little empirical evidence that course management systems actually improve pedagogy. Study findings suggest, however, that using a CMS does invite faculty to rethink their course instruction and instructional environment, resulting in a sort of accidental pedagogy ».

La plupart des études menées jusqu'à présent pour évaluer les effets des dispositifs hybrides sur la qualité des processus d'enseignement/apprentissage souffrent ainsi d'un biais commun qui pourrait expliquer, en partie, l'absence de résultat observé : nombre d'entre elles ont une portée limitée dans la mesure où elles se sont contentées d'envisager les dispositifs hybrides sans en distinguer leurs caractéristiques pédagogiques, organisationnelles ou matérielles. Il existe cependant des recherches exploratoires qui ont permis de mettre en évidence, à partir de différentes caractéristiques de ces dispositifs, un

Typologie des dispositifs hybrides de formation

certain nombre d'effets, notamment sur les apprentissages vécus par les participants, sur leur dynamique identitaire, les interactions sociales ou encore l'émergence de communauté de pratique. Charlier et Denis (2002) par exemple, ont montré l'influence du dispositif sur la capacité des apprenants à transférer les compétences acquises dans leur vie professionnelle et leurs projets personnels. Charlier, Nizet et Van Dam (2006) ont mis en évidence certains aspects du dispositif susceptibles de contribuer au développement identitaire des participants, notamment la relation entre les caractéristiques du dispositif et leurs pratiques professionnelles de référence. De plus, Deschryver (2008) identifie des conditions de l'expérience d'apprentissage en profondeur des étudiants dans des dispositifs hybrides et les conditions de gestion des 
interactions sociales dans cette perspective. Peraya et Campion (2007a et 2007b) quant à eux, ont pu montrer l'effet d'un changement de l'environnement - le passage d'un site Web à la plateforme Claroline - sur la réalisation des tâches et des travaux demandés aux étudiants, sur le comportement des groupes de travail ainsi que sur la fréquence d'utilisation des outils assurant les fonctions de collaboration et de mutualisation.

D’autres recherches entreprises récemment sur les perceptions des étudiants quant aux apprentissages effectués montrent une très nette dépendance de ces dernières en fonction de la nature du dispositif pédagogique mis en place. Ainsi les pourcentages d'accords sur des propositions exprimant, pour différentes facettes des apprentissages (Lebrun, 2005), un impact positif des TIC passent d'environ $20 \%$ pour des dispositifs de nature essentiellement transmissive à environ $60 \%$ pour des dispositifs qui intègrent des méthodes davantage actives et interactives (Docq, Lebrun et Smidts, 2008 ; Lebrun, Docq et Smidts, 2009). Ces résultats encourageants sont nuancés par les perceptions des TIC (considérées soit comme un appui ou un moteur d'évolution pédagogique, soit comme un obstacle ou un frein à cette dernière). En ce qui concerne l'effet catalyseur des TIC pour le développement professionnel des enseignants, des recherches ont montré une migration, au cours du temps, de leurs usages pédagogiques vers des formes davantage centrées sur «l'étudiant apprenant ». Il est cependant difficile de savoir s'il s'agit d'un effet « naturel », les technologies entrant de plus en plus dans les habitudes, ou s'il s'agit d'un effet des différentes mesures mises en place dans le cadre de la formation des enseignants, de l'évaluation institutionnelle des enseignements ou du soutien et de la valorisation des efforts pédagogiques des enseignants (Lebrun et al., 2009, op.cit.).

Ces premiers résultats, bien que parcellaires, montrent cependant l'importance des dimensions caractéristiques des dispositifs de formation pour en mesurer les effets sur le processus d'enseignement-apprentissage. Nous pensons donc qu'il sera d'autant plus aisé d'identifier les effets de ces dispositifs que leur description sera de qualité.

Aussi est-il nécessaire dans un premier temps de notre recherche d'élaborer un cadre descriptif validé empiriquement pour, ensuite, en comprendre et en différencier les effets. C'est dans cette perspective compréhensive que cet article propose d'établir une typologie des dispositifs hybrides de formation au départ de leurs caractéristiques pédagogiques, organisationnelles et matérielles.

\section{Méthodologie}

\section{Cadre de référence}

La première étape de la recherche a été consacrée à l'élaboration d'un cadre de référence permettant de caractériser les dispositifs hybrides de formation (Deschryver, Lameul, Peraya, Villiot-Leclercq, 2011). Ce cadre de référence s'appuie sur les travaux de Charlier, Deschryver et Peraya dont une première synthèse a fait l'objet d'une publication dans un précédent numéro de Distances et savoirs (2006). Il propose cinq dimensions principales pour la description de ces dispositifs (tableau 1) : la mise à distance et les modalités d'articulation des phases présentielles et distantes (1), l'accompagnement humain (2), les formes particulières de médiatisation (3) et de médiation (4) liées à l'utilisation d'un environnement technopédagogique et le degré d'ouverture du dispositif (5).

L'articulation entre les moments présentiels et à distance est caractérisée par le temps accordé à l'un et l'autre mode, à leur répartition ainsi qu'au type d'activités prévues et scénarisées par l'enseignant dans chacune des phases : des activités de prise ou de traitement d'information, chacune prenant plus ou moins d'importance en fonction de l'approche pédagogique adoptée, transmissive, individualiste ou encore collaborative (Charlier, Bonamy et Saunders, 2003). Chacune de ces approches se caractérise par certaines options des formateurs et concepteurs d'un dispositif concernant : le statut accordé aux connaissances, la représentation de l'apprentissage, la représentation du but de l'éducation, les choix laissés aux apprenants, la structure du cours, les critères mobilisés pour évaluer l'efficacité des apprentissages, le rôle accordé aux apprenants et le rôle du tuteur. En particulier, le statut accordé aux connaissances et aux rôles des apprenants dans leur construction paraît particulièrement déterminant. Ainsi, selon que leurs postures professionnelles

(Lameul, 2008) privilégient la transmission du savoir, sa construction dans une alternance théorie-pratique, le processus cognitif, le développement personnel ou la transformation de la société, leurs options en matière de conception des scénarios pédagogiques et d'animation des dispositifs vont probablement varier.

La notion d'accompagnement se réfère aux travaux sur le support à l'apprentissage et plus particulièrement l'accompagnement humain. Trois composantes de l'accompagnement des étudiants se retrouvent fréquemment dans la littérature sur le tutorat en formation à distance, et participent à la qualité de l'expérience d'apprentissage des étudiants : les composantes cognitive, affective et métacognitive (Audet, 2006 ; Bernatchez, 2003 ; Dionne Mercier et Deschênes, 1999). Nous avons donc choisi de nous fonder sur ces trois dimensions pour caractériser l'accompagnement. Dans sa dimension cognitive, celui-ci concerne les modalités d'accompagnement destinées à soutenir la construction de connaissances (soutien au traitement de l'information et à la réalisation des activités/méthodologique). 
L'accompagnement affectif intègre les modalités de soutien à l'engagement de l'apprenant. Il comprend la présence sociale, qui sous-entend deux dimensions : le degré d'intimité (comment je me sens proche de celui ou ceux avec qui j'apprends) et le degré de réactivité (est-ce que je reçois un feedback suffisamment rapide à mes interventions ?). Enfin, l'accompagnement métacognitif vise la construction de connaissances par une démarche réflexive sur ses propres processus cognitifs.

L'environnement technopédagogique constitue un des éléments essentiels du dispositif hybride de formation. Il repose sur des formes complexes de médiatisation et de médiation (Meunier et Peraya, 1993 et 2004). La médiatisation relève de l'ingénierie de la formation et du design pédagogique. Elle concerne les processus de conception, de production et de mise en œuvre des dispositifs, processus dans lequel le choix des médias les plus adaptés ainsi que la scénarisation occupent une place importante (Peraya, 2010). Les environnements technopédagogiques permettent la médiatisation des contenus d'apprentissage et de ressources ainsi que des fonctions génériques du dispositif de formation (Paquette, 1993 ; Collins, Greeno et al., 1994 ; Basque et Doré, 1998 ; Peraya 2000 ; de Vries, 2001 ; Henri et Lundgren-Cayrol, 2001 ; Gauthier, 2004). Celles-ci regroupent les activités menées par les acteurs mettant en œuvre des objets d'apprentissage (notamment des ressources) (Merill, 2000 ; Wiley, 2002 ; Pernin, 2003 ; Robertson, 2006) et s'appuyant sur des « services » (Gauthier, 2004), le plus souvent désignés dans la littérature comme des outils.

La médiation se définit comme le processus de transformation que produit sur les comportements humains (par exemple cognitifs ou relationnels), le dispositif technique, "l'instrument » (autrement dit un artefact technique et ses schèmes sociaux d'utilisation), à travers lequel le sujet interagit avec le monde, avec des « objets », d'autres sujets ou encore avec lui-même (Rabardel et Samurçay, 2001). Autrement dit, le dispositif technopédagogique par sa position d'intermédiation modifie le rapport du sujet au savoir, à l'action, aux autres, etc., mais il contribue aussi à transformer le savoir, l'action ainsi que la relation. L'analyse des différentes formes de médiation relève donc de l'analyse des effets. Notons que cette analyse doit tenir compte des effets escomptés, des médiations prévues par le concepteur autant que celles réellement actualisées dans l'appropriation et l'usage de l'environnement technopédagogique par les différents acteurs.

L'articulation de deux cadres convergents (sémiopragmatique de la communication médiatisée d'une part, ergonomie cognitive de l'activité humaine instrumentée, d'autre part ) permet d'identifier les formes suivantes de médiation : sémiocognitive (correspond à la médiation épistémique chez Rabardel et Samurçay qui correspond à la construction de connaissances et de sens), sensorimotrice (porte sur les comportements gestuels et moteurs induits par l'instrument), praxéologique (porte sur les conditions de réalisation de l'action), relationnelle (porte sur la relation entre les sujets) et réflexive (porte sur le sujet lui-même et implique donc une dimension « méta » fondamentale pour les processus d'apprentissage).

Enfin, la cinquième dimension concerne le degré d'ouverture du dispositif, i.e. le degré de liberté de l'apprenant face aux situations d'apprentissage (Jézégou, 2008). L'auteure identifie trois modalités selon lesquelles peut se construire ce degré de liberté : l'apprenant planifie lui-même ses apprentissages, le dispositif détermine entièrement les situations d'apprentissage, les situations d'apprentissage du dispositif sont structurées conjointement par l'apprenant et par l'enseignant. Selon l'auteure, plus le degré d'ouverture du dispositif est élevé, plus les étudiants auront tendance à s'impliquer dans leurs apprentissages.

En résumé, notre cadre de référence comprend donc cinq dimensions pour la description des dispositifs hybrides de formation : l'articulation présence/distance, la médiatisation, la médiation, l'accompagnement et le degré d'ouverture. Nous posons l'hypothèse que nous aboutirons, en croisant ces caractéristiques, à identifier quelques configurations particulières (Elias et Hulin, 1993) de dispositifs pour lesquelles nous pourrons analyser des effets spécifiques sur un certain nombre de variables de l'enseignement et de l'apprentissage.

Typologie des dispositifs hybrides de formation

\section{Elaboration du questionnaire}

Sur la base de ce cadre théorique, nous avons développé un questionnaire (12 questions fermées et 12 échelles de fréquence et d'attitude), destiné aux enseignants de l'enseignement supérieur.

Des questions fermées ont été utilisées pour appréhender les facteurs contextuels qui caractérisent l'enseignant (sexe, institution d'origine, statut professionnel, expérience professionnelle), le cours dispensé (niveau d'études, type de formation, nombre d'étudiants inscrits, durée, charge de travail totale, nombre d'heures d'enseignement assurées en présence, fréquence des cours en présence) et la plateforme utilisée pour le cours.

Les échelles d'attitude et de fréquence (ainsi qu'une question fermée complémentaire relative à l'articulation présence/distance) ont, quant à elles, été utilisées pour appréhender les cinq dimensions du cadre de référence : articulation présence/distance, médiatisation, médiation, accompagnement et degré d'ouverture. 


\section{Description de l'échantillon}

Le questionnaire en ligne a été administré à 174 enseignants selon un protocole standardisé dans plus de 22 établissements d'enseignement supérieur et universitaire en Europe (Belgique, France, Luxembourg, Suisse) et au Canada (tableau 2). L'échantillon concerne un panel varié de formations (tableau 2). Il est composé de 59,4 \% d'hommes et $40,4 \%$ de femmes.

\section{Extraction des composantes}

\begin{tabular}{|c|c|c|}
\hline & $\begin{array}{l}\text { Type d'échelle } \\
\text { P/D }\end{array}$ & alpha 1. Articulation \\
\hline Comp 1 - Participation active des étudiants en présence & fréquence & 0.80 \\
\hline Comp 2 - Participation active des étudiants à distance & fréquence & 0.69 \\
\hline \multicolumn{3}{|l|}{ 2. Accompagnement } \\
\hline Comp 10 - Accompagnement méthodologique par les enseignants & fréquence & 0.86 \\
\hline Comp 11 - Accompagnement métacognitif par les enseignants & fréquence & 0.83 \\
\hline Comp 12 - Accompagnement par les étudiants & fréquences & 0.89 \\
\hline \multicolumn{3}{|l|}{ 3. Médiatisation } \\
\hline Comp 3 - Mise à disposition d'outils d'aide à l'apprentissage & fréquence & 0.81 \\
\hline $\begin{array}{l}\text { Comp } 4 \text { - Mise à disposition d'outils de gestion, de } \\
\text { communication et d'interaction }\end{array}$ & fréquence & 0.73 \\
\hline Comp 5 - Ressources sous forme multimédia & fréquence & 0.76 \\
\hline Comp 6 - Travaux sous forme multimédia & fréquence & 0.77 \\
\hline Comp 7 - Outils de communication synchrone et de collaboration & numérique & 0.70 \\
\hline
\end{tabular}

\section{Médiation}

Comp 8 - Possibilité de commentaire et d'annotation

des documents par les étudiants

Comp 9 - Objectifs réflexifs et relationnels

attitude

0.80

\section{Ouverture}

Comp 13 - Choix de liberté des méthodes pédagogiques

degré de liberté $\quad 0.86$

Comp 14 - Recours aux ressources et acteurs externes

fréquences $\quad 0.82$

Tableau 1. Composantes principales à la base de l'établissement de la typologie

Des analyses factorielles (Analyse en composantes principales avec rotation Varimax), réalisées sur les 12 échelles, ont permis d'extraire 34 composantes principales. Une sélection au départ de critères relatifs au pouvoir de discrimination et à la qualité psychométrique des composantes a été ensuite menée pour conserver in fine 14 composantes qui serviront de base à l'établissement d'une typologie (Burton, à paraître)². Le tableau 1 reprend les 14 composantes principales ainsi

\footnotetext{
${ }^{1}$. La dimension relative aux facteurs contextuels sera envisagée plus loin car elle a été appréhendée non pas par des échelles de mesure mais par des questions fermées.

2 . Une première sélection a été réalisée sur la base des valeurs de la cohérence interne de chaque composante : les composantes pour lesquelles l'alpha de Cronbach n'était pas satisfaisant ont été supprimées des analyses. Une seconde sélection a été réalisée sur la base 1. 
que les valeurs du coefficient alpha de Cronbach calculé pour chacune d'elles. Les scores factoriels centrés réduits (moyenne $=0$ et écart type $=1$ ) ont été ensuite calculés pour repérer la position de chaque dispositif sur les composantes sélectionnées. Toutes les échelles sont orientées positivement : plus le score factoriel est élevé plus la fréquence, la liberté de choix, le nombre d'éléments (nombre et types d'activités, de ressources, d'outils de communication synchrone et de collaboration utilisés) seront élevés ou l'attitude sera favorable selon le type d'échelle considéré.

\section{Composante 1. Participation active des étudiants en présence}

La première composante principale indique la fréquence des activités des étudiants lors des phases d'enseignement en présence. Elle rassemble principalement un ensemble d'activités réalisées en groupe (discussions, débats, jeux de rôle, projets, résolutions de problème, études de cas, recherche d'information, simulations, tutorat par les pairs, débriefings, régulation d'activités, occasions d'échanges informels, prises de contact et discussions) mais aussi quelques activités individuelles (exposé, démonstration, travaux et programme de lecture).

\section{Composante 2. Participation active des étudiants à distance}

La deuxième composante principale est semblable à la première si ce n'est que les fréquences des activités des étudiants ont été localisées, cette fois-ci, par rapport aux phases d'activité à distance. Elle rassemble également bon nombre d'activités de groupe (discussions, débats, jeux de rôle, projets, résolutions de problème, études de cas, recherche d'information, simulations, tutorat par les pairs) mais aussi des activités individuelles de type programmes de lecture.

\section{Composante 3. Mise à disposition d'outils d'aide à l'apprentissage}

La troisième composante principale concerne la fréquence avec laquelle les enseignants mettent à disposition des étudiants des outils d'aide à l'apprentissage dans la plateforme utilisée pour le cours. Il s'agit par exemple du tutorat ou des formes d'accompagnement, des espaces et/ou moyens de travail (éditeur de textes, de graphismes, des gestionnaires de projet, etc.), des espaces et/ou des moyens pour réfléchir sur leur manière d'apprendre (blog, journal de bord, etc.), des indicateurs de l'activité et de la présence de leurs collègues distants ainsi que des opportunités et/ou des moyens de construire leur identité numérique (se présenter, actualiser son ou ses profils).

\section{Composante 4. Mise à disposition d'outils de gestion, de communication et d'interaction}

La quatrième composante concerne la fréquence avec laquelle les enseignants mettent à disposition des étudiants des outils de gestion, de communication et d'interaction dans la plateforme utilisée pour le cours tels des espaces et/ou des moyens de communication, d'échange et de collaboration ainsi que des moyens de gestion et d'organisation (calendrier, rappel des prochaines activités et échéances, notes de travaux...).

\section{Composante 5. Ressources sous forme multimédia}

La cinquième composante indique la fréquence avec laquelle les enseignants mettent à disposition des étudiants des ressources multimédias dans la plateforme utilisée pour le cours, telles que des documents écrits intégrants beaucoup, voire une majorité des plages visuelles (tableaux, photos, schémas, représentations mathématiques), des images numérisées

Typologie des dispositifs hybrides de formation

statiques (photographies, schémas, cartes...), des documents vidéos numérisés ou multimédias ainsi que des logiciels interactifs (animations, simulations, micromondes, univers 3D, mondes virtuels ou immersifs, réalité augmentée...).

\section{Composante 6. Travaux sous forme multimédia}

La sixième composante concerne la fréquence avec laquelle les enseignants exigent des étudiants des travaux sous forme multimédia dans la plateforme utilisée pour le cours tels que des documents écrits intégrants beaucoup, voire une majorité des plages visuelles (tableaux, photos, schémas, représentations mathématiques), des images numérisées statiques (photographies, schémas, cartes...) et des documents vidéos numérisés ou multimédias.

\section{Composante 7. Outils de communication synchrone et de collaboration utilisés}

La septième composante concerne la fréquence d'usage des outils dont les enseignants se servent dans la plateforme utilisée pour le cours. Elle regroupe les outils de communication synchrone écrite (par exemple chat), de communication

de la significativité des effets $(\mathrm{p}<0.001)$ : les composantes, pour lesquelles aucun effet significatif n'a été observé a posteriori (MANOVA en fonction de la typologie issue de l'analyse de clusters), ont été également supprimées dans les analyses finales. 
synchrone orale (téléphone portable, conférence téléphonique comme skype) et des environnements complexes de collaboration à distance (son, webcam, partage de documents et d'écran, chat, tableau blanc partagé...).

Composante 8. Possibilité de commentaire et d'annotation des documents par les étudiants

La huitième composante indique avec quelle fréquence les étudiants ont la possibilité de commenter et d'annoter les documents placés sur la plateforme utilisée pour le cours (ressources et contenus numériques, journaux de bord, blogs ou tout autre production ou travail personnel réalisé par leurs collègues).

\section{Composante 9. Objectifs réflexifs et relationnels}

La neuvième composante concerne les représentations des enseignants quant à l'utilité de la plateforme pour que les étudiants atteignent des objectifs réflexifs et relationnels tels que mieux se connaître, prendre de la distance, critiquer des savoirs ou des méthodes, développer leur confiance en soi, entrer en relation avec d'autres (étudiants, experts, groupes...) et mieux connaître la manière dont-ils apprennent.

\section{Composante 10. Accompagnement méthodologique par les enseignants}

La dixième composante figure la fréquence avec laquelle l'enseignant (ou son assistant) propose un accompagnement méthodologique. Dans cette composante, interviennent des comportements tels que favoriser la collaboration et les échanges entre étudiants (partage de ressources, mise en commun des travaux, ...), aider les groupes d'étudiants pour s'organiser, se répartir les tâches, prendre des décisions, résoudre des conflits, favoriser les échanges (se présenter, exprimer ses attentes et ses projets, ...) permettant des contacts personnalisés entre les étudiants et organiser des moments de régulation avec les groupes d'étudiants.

\section{Composante 11. Accompagnement métacognitif par les enseignants}

La onzième composante représente la fréquence avec laquelle l'enseignant (ou son assistant) propose un accompagnement métacognitif. Dans cette composante, interviennent des comportements tels que solliciter une réflexion de l'apprenant sur sa manière d'apprendre et ses résultats (journal de bord, choix de stratégies, etc.), aider les étudiants à concevoir leurs propres méthodes d'étude et solliciter une réflexion de l'apprenant sur ses acquis et/ou transfert d'apprentissage.

\section{Composante 12. Accompagnement par les étudiants}

La douzième composante représente la fréquence avec laquelle les étudiants assurent un accompagnement de leurs pairs. Dans cette composante, interviennent des comportements tels que fournir des ressources d'information à leurs pairs (explications, exemples, listes de lecture, références, experts...), présenter un résumé des cours précédents à leurs pairs, répondre à des questions de leurs pairs, faciliter les échanges entre pairs, apporter un soutien technique lié aux outils technologiques utilisés dans le cours (réponse aux questions des autres étudiants), prendre en charge le groupe collaboratif pour s'organiser, se répartir les tâches, prendre des décisions, résoudre des conflits ainsi qu'établir un contact personnalisé avec leurs pairs (se présenter, interagir pour affiner ce contact).

Composante 13. Choix de liberté des méthodes pédagogiques

La treizième composante indique le degré de liberté avec lequel les étudiants ont la possibilité d'effectuer des choix quant aux méthodes pédagogiques utilisées dans le cours telles que les cheminements possibles au sein du dispositif de formation, les formats d'interaction, les méthodes pédagogiques, la manière d'apprendre, les personnes ressources à solliciter pour être accompagné dans ses apprentissages et les moyens techniques.

\section{Composante 14. Recours aux ressources et acteurs externes}

Enfin, la quatorzième composante concerne la fréquence avec laquelle le cours fait appel à des ressources et des acteurs externes au monde académique (socioprofessionnel, famille, culture...) pour les ressources (documents, vidéos...), les situations problèmes, les cas, les apports des étudiants ainsi que pour les interventions d'experts, de praticiens ou d'acteurs. 


\section{Classification et caractérisation des groupes ${ }^{3}$}

Les données récoltées ont permis d'effectuer une analyse de clusters sur les 14 composantes principales pour créer des groupes de dispositifs hybrides. Trois méthodes différentes (Ward, lien complet, lien moyen) ont été utilisées à titre de vérification pour s'assurer de la consistance de la classification. Les corrélations enregistrées pour ces trois méthodes s'élèvent respectivement à $0.72(\mathrm{p}=0.000)$ entre la solution obtenue par la méthode de Ward et par la méthode du lien moyen, à $0.73(\mathrm{p}=0.000)$ entre la solution obtenue par la méthode du lien complet et par la méthode du lien moyen et à $0.81(\mathrm{p}=0.000)$ entre la solution obtenue par la méthode de Ward et par la méthode du lien complet. Ceci témoigne d'une réelle structuration des données initiales. La méthode Ward a été choisie dans la mesure où les méthodes du lien moyen et du lien complet favorisent respectivement l'effet de chaînage (peut empêcher la distinction de 2 groupes pourtant bien séparés) et l'effet de dissection (peut affecter à des groupes différents des objets qui sont très proches). La solution optimale et finale a été obtenue par la méthode de Ward en définissant 7 groupes, cette partition donnant lieu à la diminution du $\mathrm{R}^{2}$ (rapport de la somme des carrés des écarts entre les groupes et de la somme des carrés des écarts totale) la plus importante. Les groupes constitués par la méthode de Ward sont homogènes (dispositifs les plus similaires possibles au sein des types) et différenciés (types aussi dissemblables que possible).

Pour pouvoir caractériser les groupes obtenus en vue de créer une typologie, une MANOVA a été réalisée au départ des 14 composantes principales en prenant comme critère de classification la solution obtenue lors de l'analyse de cluster selon la méthode de Ward. Les statistiques descriptives ont ensuite été calculées pour chaque type sur chacune des 14 composantes principales suivant les scores factoriels standardisés.

Des analyses de correspondances ont été réalisées pour exploiter les réponses des enseignants aux questions fermées en prenant comme seconde variable la solution obtenue lors de l'analyse de cluster selon la méthode de Ward. Ces variables n'ont donc pas servi directement à l'élaboration des types mais ont permis de caractériser a postériori la typologie obtenue.

\section{Résultats}

Les résultats seront présentés sous trois formes. Dans une première approche, des représentations graphiques sous forme de radars donneront la position de chaque type de dispositifs par rapport aux 14 composantes principales retenues pour la description de la typologie. Cette première approche se veut purement descriptive et procède sans tenir compte des caractéristiques des autres types. Elle a pour objectif d'établir une première description de la typologie indépendamment des rapprochements ou des distinctions que l'on peut établir entre les types. Ensuite, un tableau synthétique sera présenté pour procéder à une comparaison des différents types. Enfin, les analyses de correspondance permettront de compléter les définitions établies en examinant les relations entre les types de dispositifs et diverses variables contextuelles.

Typologie des dispositifs hybrides de formation

Ces différentes analyses nous permettent de proposer une organisation des 6 types de dispositifs ${ }^{4}$ retenus allant du dispositif le moins développé au plus développé comme présenté ci-dessous :

- type 1. Dispositif centré enseignement et acquisition de connaissances,

- type 2. Dispositif centré enseignement mettant à disposition des ressources multimédias,

- type 3. Dispositif centré enseignement mettant à disposition des outils d'interaction,

- type 4. Dispositif centré enseignement tendant vers le support à l'apprentissage,

- type 5. Dispositif ouvert centré apprentissage,

- type 6. Dispositif ouvert centré apprentissage soutenu par un environnement riche et varié.

\section{Définition des types de dispositifs}

La première interprétation des résultats consiste donc à établir une première description de chaque type en les positionnant par rapport aux 14 composantes. Pour ce faire, des graphiques sous forme de radars ont été employés. Dans ces graphiques, les valeurs indiquées représentent les scores factoriels standardisés (moyenne $=0$ et écart-type $=1$ ) issus

\footnotetext{
${ }^{3}$. Les analyses décrites dans cette section ont été effectuées initialement au départ de l'ensemble des 34 composantes extraites du questionnaire. Ces analyses ont ensuite été reconduites sur les 14 composantes sélectionnées sur base des résultats de la première MANOVA. Ce sont ces dernières qui sont présentées ici.
} 
des analyses factorielles. Pour rappel, la MANOVA effectuée sur ces 14 composantes sélectionnées en fonction des types donne lieu à des effets très hautement significatifs $(\mathrm{p}<0.001)$. Nous obtenons de la sorte les définitions des 6 types suivants.

\section{Type 1. Dispositif centré enseignement et acquisition de connaissances}

Graphique 1. Scores factoriels du type 1 selon les 14 composantes

Le type 1 représente $19 \%$ des dispositifs envisagés dans l'étude.

Articulation présence/distance. Il s'agit du type de dispositif où les étudiants participent le plus rarement voire jamais de manière active au processus d'enseignement que ce soit en présence (comp1) ou à distance (comp2).

Médiatisation. Les ressources mises à disposition par les enseignants (comp5) ainsi que les travaux exigés des étudiants (comp6) sont très rarement voire jamais sous forme multimédia. Il s'agit du dispositif où les outils technologiques de gestion, de communication et d'interaction (comp4) sont le moins souvent mis à disposition. De même, quasiment aucun outil de communication synchrone et de collaboration n'est utilisé dans la plateforme pour le cours (comp7). Les outils d'aide à l'apprentissage (comp3) ne sont quant à eux quasiment jamais mis à disposition sur la plateforme.

4. Initialement, 7 types de dispositifs ont été identifiés par analyse de clusters. Un type a cependant été exclu des résultats finaux dans la mesure où il comprenait 3 dispositifs aux caractéristiques atypiques et très variées (voir ci-dessous).

Médiation. Les enseignants appartenant à ce type affichent clairement des avis très négatifs quant aux objectifs relationnels et réflexifs (comp9) attendus des usages et des outils de l'environnement technologique. Les étudiants n'ont quasiment jamais la possibilité de commenter et d'annoter les documents placés sur la plateforme (comp8).

Accompagnement. L'accompagnement de l'apprentissage (tutorat (comp12), accompagnement métacognitif (comp11) et méthodologique (comp10) ne sont rarement voire jamais organisés.

Ouverture. Il n'offre quasiment aucune possibilité de choix dans les méthodes pédagogiques (comp13). L'enseignant n'utilise que très rarement des ressources ou acteurs externes au monde académique (comp14). Il s'agit du dispositif le plus fermé. 


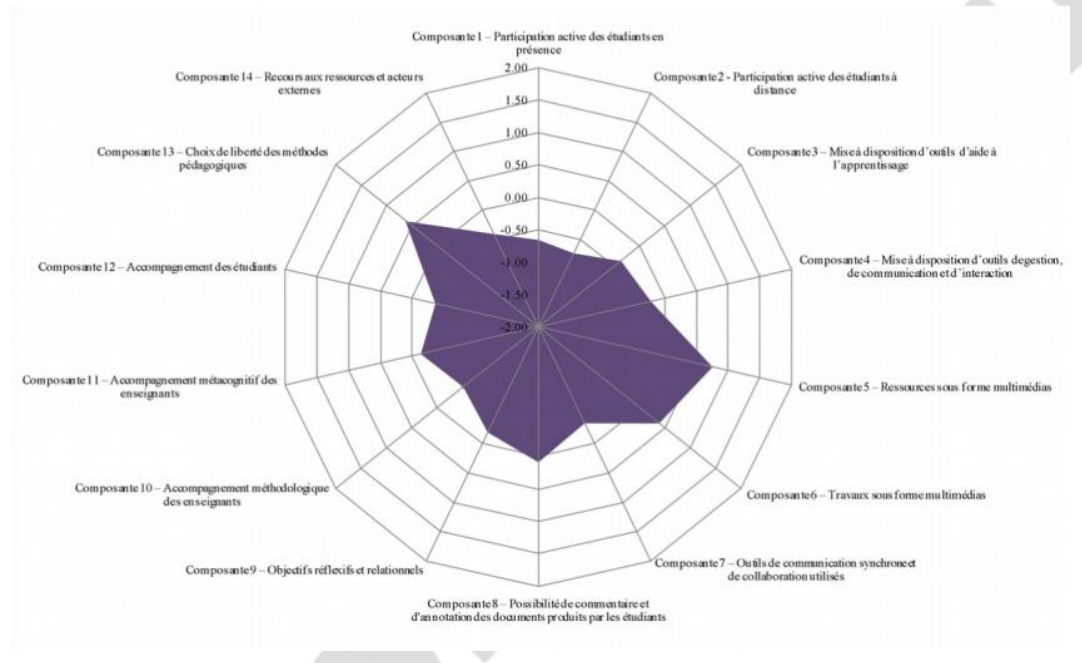

Graphique 2. Scores factoriels du type 2 selon les 14 composantes

Le type 2 représente $18 \%$ des dispositifs envisagés dans l'étude.

Articulation présence/distance. Il s'agit d'un type de dispositif où les étudiants participent le plus rarement de manière active au processus d'enseignement que ce soit en présence (comp1) ou à distance (comp2).

Médiatisation. L'enseignant fournit assez souvent des ressources sous forme multimédia (comp5). Il compte parmi les dispositifs les plus développés sur ce point. Par contre, il s'agit d'un des types de dispositifs où les outils technologiques de gestion, de communication et d'interaction (comp4) sont moins souvent mis à disposition. Quasiment aucun outil de communication synchrone et de collaboration n'est utilisé dans la plateforme utilisée pour le cours (comp7). Les outils d'aide à l'apprentissage (comp3) sont quant à eux rarement mis à disposition sur la plateforme. Les travaux demandés aux étudiants sont parfois à dominante multimédias (comp6).

Médiation. Les avis des enseignants appartenant à ce type sont très contrastés quant aux objectifs relationnels et réflexifs (comp9) attendus des usages et des outils de l'environnement technologique. Les étudiants ont également rarement la possibilité de commenter et d'annoter les documents placés sur la plateforme (comp8).

Accompagnement. L'accompagnement de l'apprentissage sous forme de tutorat (comp12) et méthodologique (comp10) est rarement organisé. L'accompagnement métacognitif est, quant à lui, parfois pris en charge par les enseignants.

Ouverture. Le type 2 présente une situation contrastée entre des dispositifs offrant une faible à grande liberté de choix au niveau des méthodes pédagogiques. L'enseignant a parfois recourt à des ressources ou acteurs externes au monde académique. 
Type 3. Dispositif centré enseignement mettant à disposition des outils d'interaction

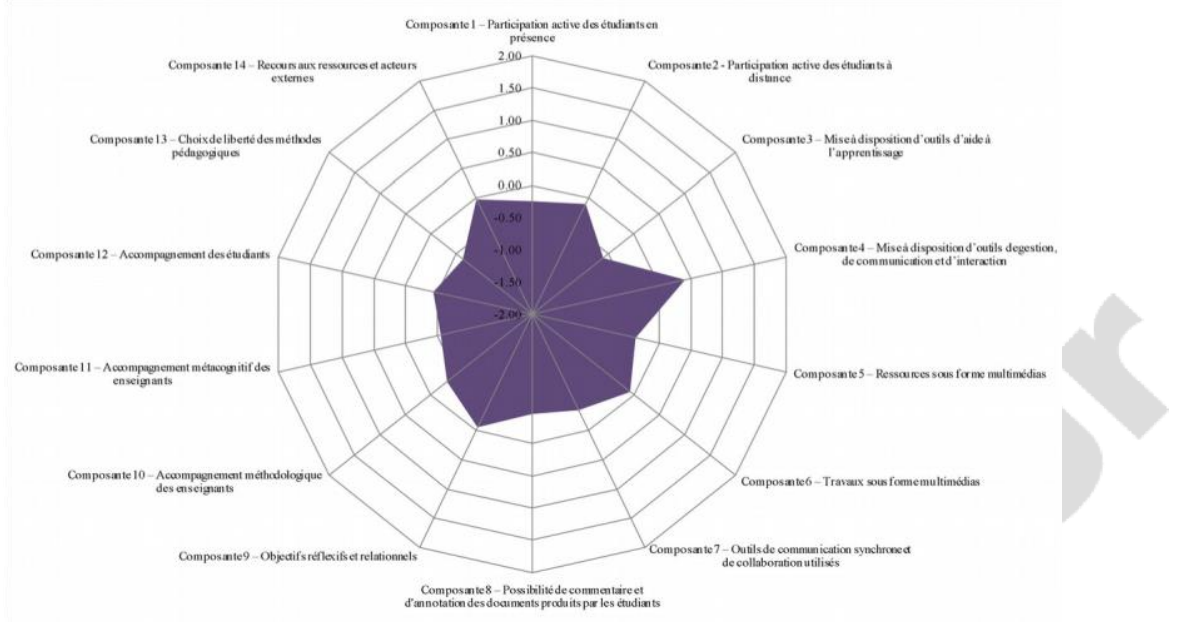

Graphique 3. Scores factoriels du type 3 selon les 14 composantes

Le type 3 représente $21 \%$ des dispositifs envisagés dans l'étude.

Articulation présence/distance. Les étudiants ne participent activement au processus d'enseignement que de manière épisodique (c'est-à-dire parfois) que ce soit en présence (comp1) ou à distance (comp2).

Médiatisation. Les outils technologiques mis à disposition sont plutôt destinés à la gestion de l'enseignement (comp4) qu'à l'aide à l'apprentissage (comp3). L'enseignant fournit parfois les ressources sous forme de documents écrits avec plages visuelles (comp5). Les travaux demandés aux étudiants sont rarement à dominante multimédias (comp6). Quasiment aucun outil de communication synchrone et de collaboration n'est utilisé dans la plateforme qui sert de support au cours (comp7).

Médiation. Les avis des enseignants appartenant à ce type sont très contrastés quant aux objectifs relationnels et réflexifs (comp9) attendus des usages et des outils de l'environnement technologique. Ils ne permettent pas de dégager une tendance dominante. Les étudiants ont rarement voire jamais la possibilité de commenter et d'annoter les documents placés sur la plateforme (comp8).

Accompagnement. L'accompagnement de l'apprentissage (tutorat (comp12), accompagnement métacognitif (comp11) et méthodologique (comp10) est rarement organisé et serait laissé assez souvent à l'initiative des étudiants à travers la mise à disposition d'outils de gestion, de communication et d'interaction (comp4).

Ouverture. Il n'offre quasiment aucune possibilité de choix dans les méthodes pédagogiques (comp13). L'enseignant utilise quelquefois des ressources ou acteurs externes au monde académique (comp14). Il peut donc être considéré comme relativement fermé. 
Type 4. Dispositif centré enseignement tendant vers le support à l'apprentissage

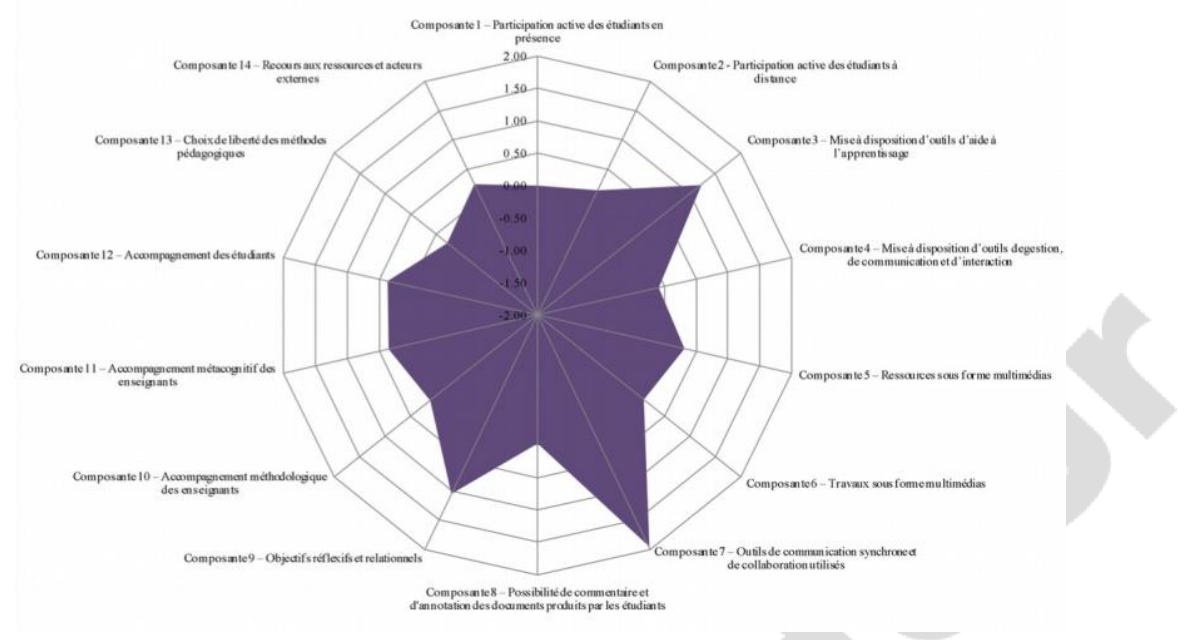

Graphique 4. Scores factoriels du type 4 selon les 14 composantes

Le type 4 représente $8 \%$ des dispositifs envisagés dans l'étude.

Articulation présence/distance.Les étudiants participent parfois de manière active au processus d'enseignement que ce soit en présence (comp1) ou à distance (comp2).

Médiatisation. Les enseignants mettent souvent à disposition des étudiants des outils d'aide à l'apprentissage (comp3) ainsi que des outils de gestion, de communication et d'interaction (comp4). Les ressources mises à disposition par les enseignants prennent assez souvent une forme multimédia (comp5) tandis que les travaux exigés des étudiants (comp6) ne prennent que parfois cette forme. Dans la plateforme utilisée pour le cours, beaucoup d'outils de communication synchrone et de collaboration sont utilisés (comp7).

Médiation. Les étudiants ont parfois la possibilité de commenter et d'annoter les documents placés sur la plateforme (comp8). C'est dans ce type, que les enseignants montrent les avis les plus favorables envers les objectifs relationnels et réflexifs (comp9) attendus des usages et des outils de l'environnement technologique.

Accompagnement. Les accompagnements méthodologique (comp10) et métacognitif (comp11) sont respectivement assez souvent et souvent organisés par les enseignants tandis que les tutorats (comp12) sont assez souvent assurés par les étudiants exactement comme dans le type 5.

Ouverture. Il offre une faible liberté de choix dans les méthodes pédagogiques (comp13) et n'a que parfois recours aux ressources ou acteurs externes au monde académique (comp14). Il s'agit d'un dispositif relativement fermé. 


\section{Type 5. Dispositif ouvert centré apprentissage}

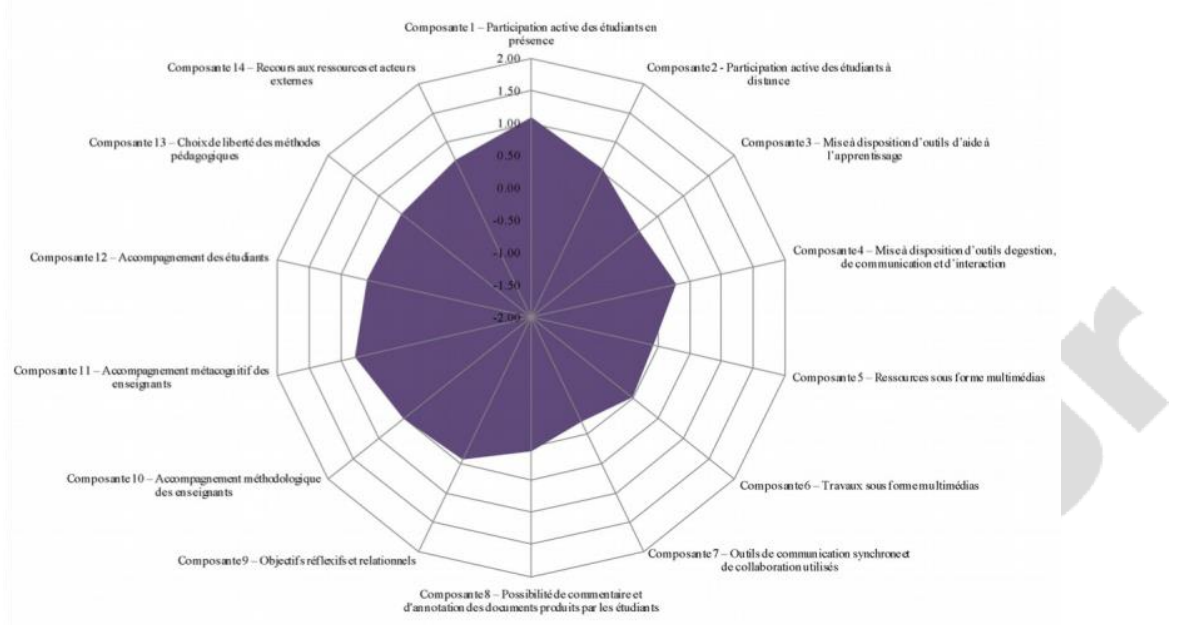

Graphique 5. Scores factoriels du type 5 selon les 14 composantes

Le type 5 représente $24 \%$ des dispositifs envisagés dans l'étude.

Articulation présence/distance. Les étudiants participent assez souvent de manière active au processus d'enseignement que ce soit en présence (comp1) ou à distance (comp2).

Médiatisation. Les enseignants mettent, sur la plateforme utilisée pour le cours, rarement à disposition des étudiants des outils d'aide à l'apprentissage (comp3) mais assez souvent des outils de gestion, de communication et d'interaction (comp4). Les ressources mises à disposition par les enseignants (comp5) ainsi que les travaux exigés des étudiants (comp6) sont parfois sous forme multimédia. Quasiment aucun outil de communication synchrone et de collaboration n'est utilisé (comp7).

Médiation. Les étudiants ont rarement la possibilité de commenter et d'annoter les documents placés sur la plateforme (comp8). Les enseignants appartenant à ce type affichent des avis partagés quant aux objectifs relationnels et réflexifs (comp9) attendus des usages et des outils de l'environnement technologique.

Accompagnement.Les accompagnements méthodologique (comp10) et métacognitif (comp11) sont respectivement assez souvent et souvent organisés par les enseignants tandis que les tutorats (comp12) sont assez souvent assurés par les étudiants.

Ouverture. Il offre une assez grande liberté de choix dans les méthodes pédagogiques (comp13) et a souvent recours aux ressources ou acteurs externes au monde académique (comp14). Il s'agit d'un dispositif des plus ouverts. 
Type 6. Dispositif ouvert centré apprentissage soutenu par un environnement riche et varié

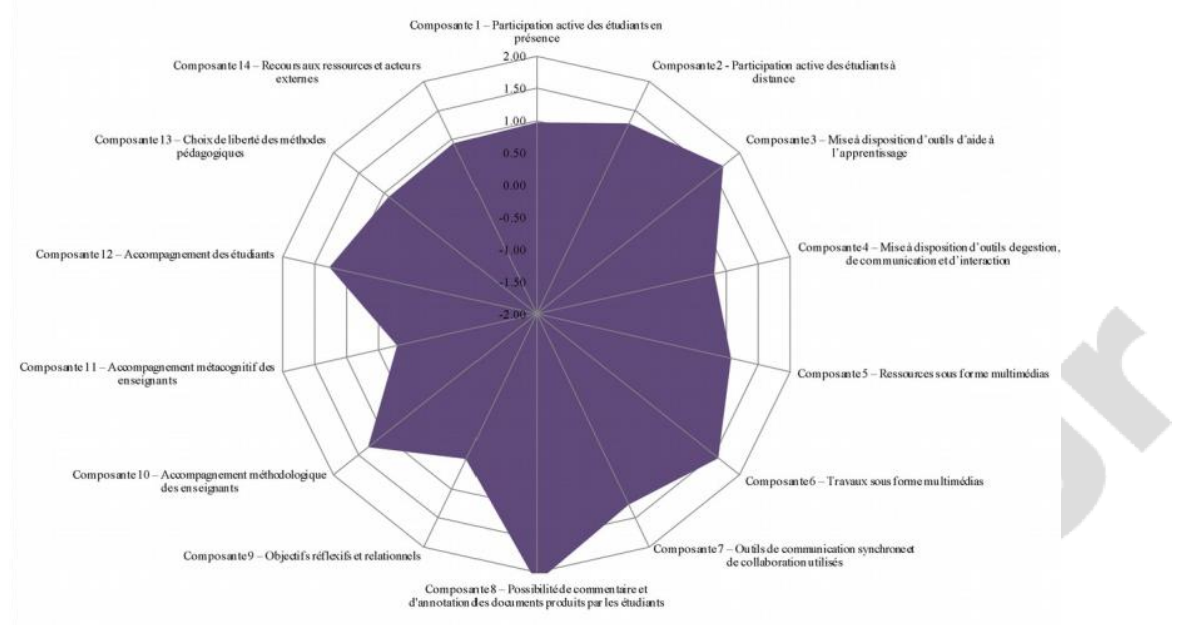

Graphique 6. Scores factoriels du type 6 selon les 14 composantes

Le type 6 représente $7 \%$ des dispositifs envisagés dans l'étude.

Articulation présence/distance. Il s'agit du type de dispositif où les étudiants participent le plus souvent de manière active au processus d'enseignement que ce soit en présence (comp1) ou à distance (comp2).

Médiatisation. Les enseignants mettent souvent à disposition des étudiants des outils d'aide à l'apprentissage (comp3) et très souvent des outils de gestion, de communication et d'interaction (comp4). Les ressources mises à disposition par les enseignants et les travaux exigés des étudiants (comp6) se présentent souvent sous forme multimédia (comp5). Dans la plateforme utilisée pour le cours, beaucoup d'outils de communication synchrone et de collaboration sont utilisés (comp7).

Médiation. Il s'agit du dispositif où les étudiants ont le plus souvent la possibilité de commenter et d'annoter les documents placés sur la plateforme (comp8). Les avis des enseignants sont également favorables envers les objectifs relationnels et réflexifs (comp9) attendus des usages et des outils de l'environnement technologique.

Accompagnement. L'accompagnement méthodologique et métacognitif est souvent organisé par l'enseignant tout comme les tutorats assurés par les étudiants. Il s'agit du type de dispositif où l'accompagnement est le plus développé.

Ouverture. Il offre une assez grande liberté de choix dans les méthodes pédagogiques (comp13) et a souvent recours aux ressources ou acteurs externes au monde académique (comp14).

\section{Comparaison des six types de dispositifs hybrides entre eux}

Un tableau synthétique (tableau 2 ) reprend les caractéristiques de chaque type sous forme de symboles. Ces symboles ont été attribués en fonction de scores bruts (moyenne des réponses aux items entrant dans la définition de la composante). Pour les échelles de fréquences, les scores de $0,1,2,3$ ont été respectivement attribués aux modalités « jamais », « parfois », « souvent », « très souvent ». Les symboles ---, --, -, +, ++, +++ traduisent la position de l'intervalle de confiance de la moyenne des scores bruts de selon le schéma suivant: 


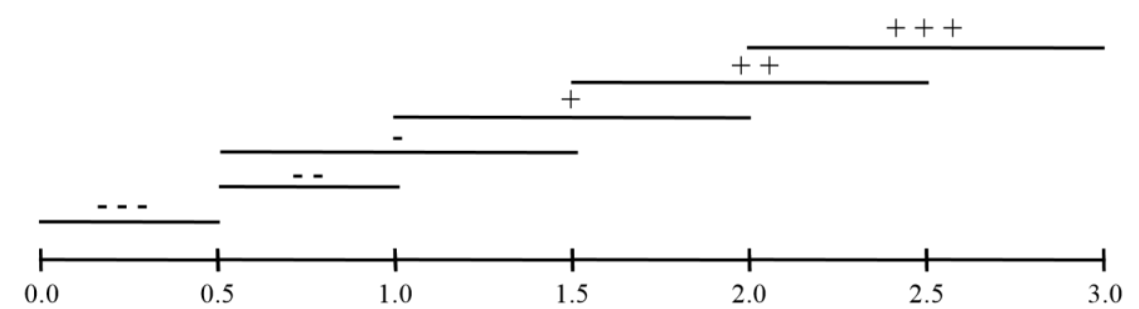

Tableau 2. Comparaison des types de dispositifs hybrides

\begin{tabular}{|c|c|c|c|c|c|c|c|c|}
\hline & & & Type 1 & Type 2 & Type 3 & Type 4 & Type 5 & Type 6 \\
\hline \multirow{2}{*}{ 产 } & Comp 1 & Participation active des étudiants en présence & -- & -- & - & - & + & ++ \\
\hline & Comp 2 & Participation active des étudiants à distance & --- & -- & - & - & + & ++ \\
\hline \multirow{5}{*}{ 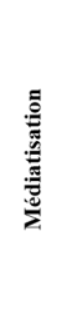 } & Comp 3 & Mise à disposition d'outils d'aide à l'apprentissage & --- & -- & -- & ++ & -- & ++ \\
\hline & Comp 4 & $\begin{array}{l}\text { Mise à disposition d'outils de gestion, de communication et } \\
\text { d'interaction }\end{array}$ & -- & - & + & ++ & + & +++ \\
\hline & Comp 5 & Ressources sous forme multimédia & -- & + & - & + & - & ++ \\
\hline & Comp 6 & Travaux sous forme multimédia & -- & - & -- & - & - & ++ \\
\hline & Comp 7 & Outils de communication synchrone et de collaboration utilisés & -- & -- & -- & ++ & --- & ++ \\
\hline \multirow{2}{*}{ 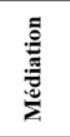 } & Comp 8 & Possibilité de commentaire et d'annotation des documents & -- & -- & -- & - & -- & ++ \\
\hline & Comp 9 & Objectifs réflexifs et relationnels & -- & - & + & ++ & + & ++ \\
\hline \multirow{3}{*}{ 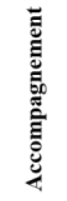 } & Comp 10 & Accompagnement méthodologique par les enseignants & -- & -- & -- & + & + & ++ \\
\hline & Comp 11 & Accompagnement métacognitif par les enseignants & -- & - & -- & ++ & ++ & ++ \\
\hline & Comp 12 & Accompagnement par les étudiants & -- & -- & -- & + & + & ++ \\
\hline \multirow{2}{*}{ 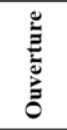 } & Comp 13 & Choix de liberté des méthodes pédagogiques & --- & + & -- & - & + & + \\
\hline & Comp 14 & Recours aux ressources et acteurs externes & -- & - & + & - & ++ & ++ \\
\hline \multicolumn{3}{|c|}{ Nombre de composantes relativement développées } & $\mathbf{0}$ & 2 & 3 & 8 & 9 & 14 \\
\hline
\end{tabular}


Une méthode analogue a été utilisée pour les autres types d'échelles. L'objectif de cette approche est de comparer les types entre eux. Le tableau 3 propose également un indice de développement des types de dispositifs hybrides qui correspond au nombre de composantes pour lesquelles le score brut se situe à cheval ou au-delà du point central de l'échelle.

Au niveau de l'articulation présence - distance, on constate que les types 1,2, 3 et 4 sont similaires et s'opposent aux types 5 et 6 où la participation active des étudiants est très développée. Les types $1,2,3$ et 5 sont en règle générale peu développés au niveau de la médiatisation et de la médiation et s'opposent ainsi aux types 4 et 6 . Dans les types 1 , 2 et 3 , l'accompagnement des étudiants est quasiment inexistant alors qu'il est très présent dans les types 4,5 et 6 . Enfin, les types $1,2,3$ et 4 sont peu ouverts par rapport aux types 5 et 6 .

\section{Relation entre les types et les variables contextuelles}

En complément des premières analyses descriptives réalisées sur la base des scores factoriels issus de l'analyse en composantes principales, des analyses de correspondance ont été menées a posteriori pour tenter de préciser les variables contextuelles intervenant dans chacun des types de dispositifs. Dans ces analyses, la première variable envisagée concernait le type de dispositif qui a été chaque fois croisé avec :

- le type d'articulation des phases d'enseignement en présence et d'activité à distance,

- l'expérience professionnelle des enseignants,

- le statut professionnel des enseignants,

- le type de formation envisagé,

- la fréquence des sessions au sein du cours,

- le niveau d'étude, - la plateforme utilisée.

Ces analyses permettent de mettre en évidence certaines conjonctions ou oppositions entre, d'une part, les types de dispositifs et, d'autre part, les modalités de réponses des enseignants aux différentes variables contextuelles.

En ce qui concerne le type d'articulation des phases d'enseignement en présence et d'activité à distance, nous avons distingué a priori, lors de l'élaboration du questionnaire, six modalités différentes :

Selon les analyses menées, nous pouvons compléter les définitions élaborées précédemment. Dans les dispositifs centrés enseignement et acquisition de connaissances (type 1), centrés enseignement mettant à disposition des ressources multimédias (type 2) et centrés enseignement mettant à disposition des outils d'interaction (type 3), l'articulation des phases d'enseignement en présence et d'activité à distance se présenterait avant tout sous la forme d'une succession de plusieurs phases d'enseignement en présence soutenues parallèlement par un dispositif continu d'activités à distances $(57,6 \%, 64,5 \%$ et 51,4\%). Dans les dispositifs ouverts centrés apprentissage (type 5), il s'agirait également de ce type d'articulation $(50,0 \%)$ ou d'une succession de plusieurs petites phases $(>3)$ d'enseignement en présence et d'activités à distance $(27,5 \%)$. Pour les dispositifs centrés enseignement tendant vers le support à l'apprentissage (type 4$)$ ainsi que pour les dispositifs ouverts centrés apprentissage soutenus par un environnement riche et varié (type 6), il n’y a pas de correspondance claire au niveau de l'articulation des phases d'enseignement en présence et d'activité à distance. Les différences de profils entre les types ne sont cependant pas significatives $\left(\square^{2}=26.611, p=0.376\right)^{4}$.

4. $\square 2$ d'indépendance. 


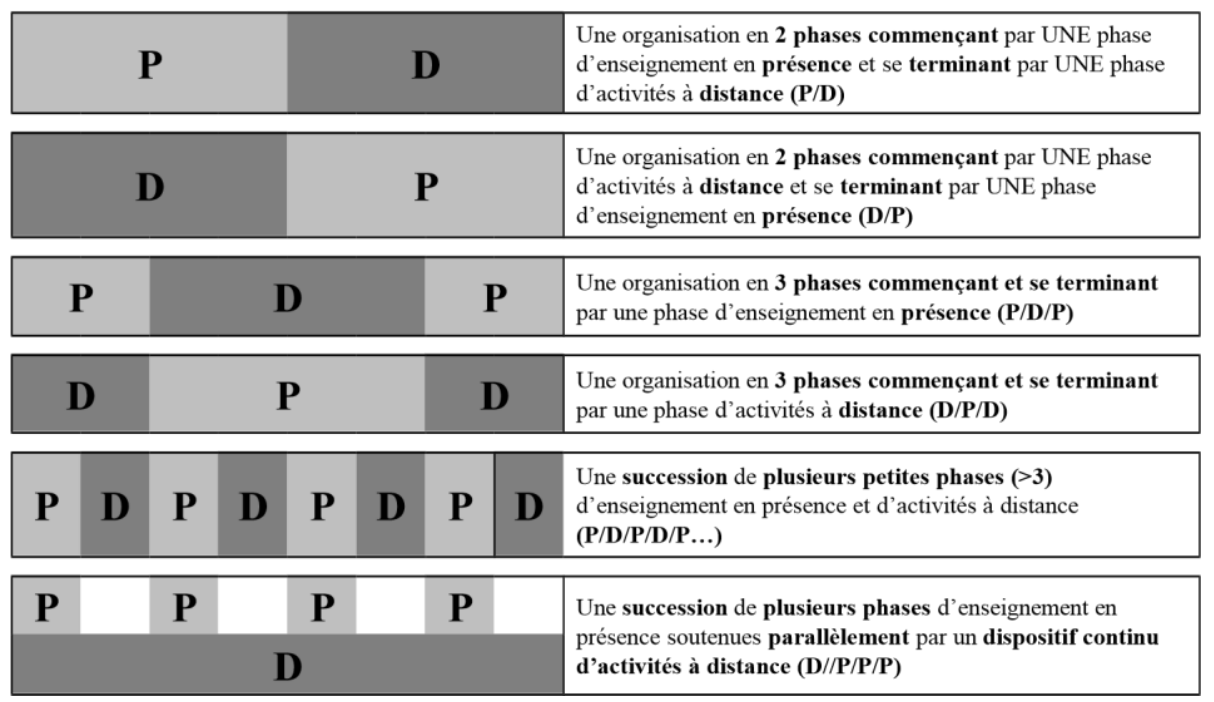

En ce qui concerne le statut de l'enseignant, les enseignants qui proposent des dispositifs de type 2 et 3 seraient avant tout des professeurs (66,7 \% et $50 \%$ ), les assistants proposant davantage des dispositifs de type 1 c'est-à-dire le type de dispositif le moins développés. Les types 4, 5 et 6 seraient quant à eux du ressort de personnes qui ont un autre statut que professeur ou assistant. Les différences de profils entre les types ne sont pas non plus significatives dans ce cas-ci $\left(\square^{2}=\right.$ $15.998, \mathrm{p}=0.100)$.

Les types 1 et 2 apparaissent en bachelor/licence (78,8 \% et 70,0 \%), 3 et 4 plutôt en master $(60,5 \%$ et $50,0 \%)$ et les types 5 et 6 à tous les niveaux d'étude $\left(\square^{2}=38.657, \mathrm{p}=0.001\right)$.

Les types 1, 2 et 3 sont avant tout des dispositifs où les cours sont dispensés chaque semaine $(87,9 \%, 83,9 \%$ et 68,4 $\%)$ et les types 4,5 et 6 sont dispensés à des fréquences diverses $\left(\square^{2}=39,220, p=0,035\right)$.

C'est dans le type 1 que l'on trouverait les enseignants qui affichent l'expérience professionnelle la plus courte ${ }^{6}(<5$ ans : 42,4\%). Rappelons que ce type de dispositifs est le moins développé par rapport aux 14 composantes qui ont servi à l'élaboration de la typologie. Dans le type 3, légèrement plus développé que le type 1 en termes de participation active des étudiants en présence et à distance, d'outils de gestion, de communication et d'interaction, d'objectifs réflexifs et relationnels et de recours aux ressources et acteurs externes au monde académique, les enseignants afficheraient majoritairement une expérience professionnelle de 5 à 10 ans (28,9\%). Le type 2 qui est également peu développé est caractérisé par une expérience professionnelle assez grande de 21 à 30 ans (32,3\%). Les types 4 et 6 qui figurent parmi les dispositifs les plus développés seraient davantage associés à des enseignants dont l'expérience professionnelle varie de 11 à 20 ans $(35,7 \%$ et 33,3\%) bien que l'on retrouve également beaucoup d'enseignants inexpérimentés dans le type $6(41,7 \%)$. Enfin dans le type 5, dispositif assez développé et ouvert, on trouverait des enseignants dont l'expérience professionnelle varie de 5 à plus de 30 ans mais les différences de profils entre les types ne sont pas significatives $\left(\square^{2}=\right.$ 26.646, $\mathrm{p}=0.146)$.

Les plateformes principalement utilisées dans le type 3 sont Caroline/i-campus (64,9\%). Dans les types 1 et 5 se partagent les plateformes Moodle (45,5 et 38,1\%) et Caroline/i-campus (30,3\% et 35,7 \%) tandis que le type 2 se partagent les plateformes Caroline/i-campus $(35,5 \%)$, Moodle $(22,6 \%)$ et Spiral $(19,4 \%)$; les types 4 et 6 étant principalement associés à Moodle $(50,0 \%$ et $53,8 \%)\left(\square^{2}=88.922, \mathrm{p}=0.000\right)$.

\section{Conclusions et perspectives}

Par rapport à la nécessité d'affiner le cadre descriptif des dispositifs pour en comprendre les effets différenciés, une typologie des dispositifs hybrides de formation a été élaborée en fonction de cinq dimensions : l'articulation présence/distance, la médiatisation, la médiation, l'accompagnement et le degré d'ouverture.

\footnotetext{
5 . Le statut réfère à la personne qui a répondu au questionnaire. Le fait que ce soit l'assistant qui réponde pourrait signifier que le professeur titulaire du cours est peu impliqué dans le développement du dispositif hybride.

${ }^{6}$. Une interprétation similaire proposée dans la note 5 peut être invoquée ici également.
} 
Au terme des analyses, la typologie semble se structurer selon le degré de développement des composantes au sein des configurations de chaque type. Celle-ci nous permettra de poser des hypothèses quant à la relation entre la qualité des apprentissages et le degré de développement des dispositifs.

Le type 1, type de dispositif le moins développé, occupe la première position de la hiérarchie tandis que le type 6 , type le plus développé, en occupe la dernière.

Dans le cadre de notre étude et selon cette hiérarchie, se trouveraient, en premier lieu trois dispositifs centrés enseignement (type 1, type 2, type 3) caractérisés par des indices d'ouverture, d'accompagnement et de participation active des étudiants relativement faibles. Ces dispositifs sont peu développés en regard des 14 composantes et principalement organisés selon une succession de plusieurs phases d'enseignement en présence soutenues parallèlement par un dispositif continu d'activités à distances.

Ce premier groupe s'oppose à un autre groupe dans lequel on retrouve des dispositifs très développés et centrés sur l'apprentissage : les types 5 et 6 . Dans ces dispositifs, les indices d'ouverture, d'accompagnement et de participation active des étudiants sont relativement élevés. Le type 5 présente cependant des caractéristiques relativement faibles en termes de médiatisation et de médiation.

Le type 4 présente à la fois des caractéristiques du premier et du second groupe : si la participation active des étudiants et le degré d'ouverture sont relativement faibles, il est très développé en termes de médiatisation et d'accompagnement.

Notons cependant qu'il s'agit d'une étude exploratoire sur un nombre limité de dispositifs. Rien ne nous dit qu'il ne serait pas possible de mettre en évidence d'autres configurations de dispositifs hybrides que celles envisagées ici si nous portions l'étude sur un plus grand échantillon. De même, bien que l'étude ait été menée dans un souci extensif, il n'en demeure pas moins que les dimensions qui y sont envisagées sont loin d'être exhaustives. La dimension relative à l'évaluation n'a, par exemple, pas été intégrée à l'étude.

Néanmoins, la typologie obtenue devrait permettre de mettre en évidence des effets différenciés des dispositifs hybrides de formation aux niveaux de la qualité des apprentissages, du développement professionnel des enseignants ainsi que sur les changements institutionnels qu'ils engendrent. Elle ouvre des pistes de recherche variées en ce qui concerne l'analyse des effets des dispositifs hybrides. Etant donné la nature des résultats obtenus, nous pourrons en effet envisager l'analyse des effets des dispositifs hybrides selon plusieurs approches. Une première approche différentielle, basée sur des comparaisons de moyennes, consisterait à évaluer ces effets en les regroupant par type et en considérant les différences entre eux. Une autre approche corrélationnelle, basée sur des modèles de régression, consisterait à positionner les dispositifs hybrides dans un espace continu selon leurs caractéristiques (composantes) et d'étudier la structure corrélationnelle de ces caractéristiques croisées avec les effets attendus. Mieux encore, une dernière approche implicative, basée sur les modèles structuraux, pourrait mettre en évidence les relations de «cause à effet » entre les caractéristiques des dispositifs hybrides et leurs effets?

De plus, ces études quantitatives seront complétées par des entretiens et par des analyses qualitatives permettant d'approfondir et de préciser les résultats des approches quantitatives mentionnées. Pour finir, des études longitudinales ou transversales pourraient être entreprises pour étudier l'évolution des dispositifs hybrides à travers le temps et l'espace.

\section{Bibliographie}

Basque J., Doré S., (1998). « Le concept d'environnement d'apprentissage informatisé », Journal of Distance Education, Revue de l'Enseignement à Distance, 13(1).

Biggs J., (1996). “Enhancing teaching through constructive alignment”, Higher Education, 32, p. 347-364.

Burton R., (à paraître). «L'évaluation des dispositifs hybrides de formation », Mesure et évaluation en éducation.

Charlier B., Denis B., (2002). «Articuler distance et présence dans une formation d'adultes en Technologie de l'Education », Colloque de l'AIPU (Association Internationale de Pédagogie Universitaire), Louvain-La-Neuve (Belgique), http://www.unifr.ch/didactic /article.php3?id_article=13

\footnotetext{
${ }^{7}$. Cet aspect fait l'objet d'une seconde étude dans le projet Hy-Sup.
} 
Charlier B., Bonamy J., Saunders M., (2003). « Apprivoiser l'innovation », B. Charlier et D. Peraya (dir.), Technologies et innovation en pédagogie. Dispositifs innovants pour l'enseignement supérieur, (43-68). Bruxelles: De Boeck.

Charlier B., Deschryver, N. et Peraya, D. (2006). « Apprendre en présence et à distance. Une définition des dispositifs hybrides ». Distances et Savoirs, 4(4), p. 469-496.

Charlier B., Nizet J. et Van Dam D. (2006). Voyage au pays de la formation des adultes : dynamiques identitaires et trajectoires sociales, Paris, L'Harmattan.

Clark R. E. (1994). “Media Will Never Influence Learning”, Educational Technology Research and Development, vol. 42, n 2, p. $21-$ 29.

Clark R. E. (1983). "Reconsidering Research on Learning from Media”, Review of Education Research, vol. 53, n 4, p. 445-459.

Collins A.-S., Greeno J. G. et Resnick L. B. (1994). "Learning environments”, T. Husen et T. N. Poslethwaite(dir.), The international encyclopedia of education, $2^{\mathrm{e}}$ éd., vol. 3, p. 3297-3302. Oxford Elsevier Science.

de Vries E. (2001). «Les logiciels d'apprentissage : panoplie ou éventail ? », Revue Française de Pédagogie, 137, p. $105-116$.

Deschryver N., Lameul G., Peraya D., et al. (2011). «Quel cadre de référence pour l'évaluation des dispositifs de formation hybrides ?», $23^{e}$ colloque de l'Adméé-Europe - Evaluation et enseignement supérieur, Paris.

Deschryver, N. (2008). Interaction sociale et apprentissage en formation hybride, Thèse de Doctorat. Genève : FPSE, Université de Genève.

Dionne M., Mercier J., Deschênes A.-J., Bilodeau H., Bourdages L., Gagné P., Lebel C. et Rada-Donath A. (1999). «Profil des activités d'encadrement comme soutien à l'apprentissage en formation à distance », Revue Distances, 13(2), http://cqfd.teluq. uquebec.ca/distances/D3_2_e.pdf

Docq F., Lebrun M. et Smidts D. (2008). « À la recherche des effets d'une plate-forme d'enseignement/apprentissage en ligne sur les pratiques pédagogiques d'une université : premières approches ", Revue internationale des technologies en pédagogie universitaire, 5(1), p. 45-57. http://www.ritpu.org/IMG/pdf/RITPU_v05n01_45.pdf

Elias N. et Hulin, M. (dir.) (1993). Engagement et distanciation : contributions à la sociologie de la connaissance, Paris, Fayard.

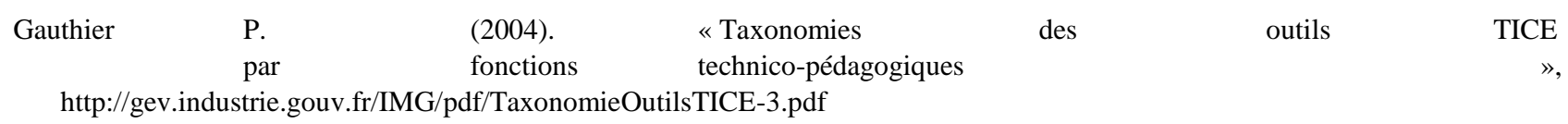

Henri F. et Lundgren-Cayrol K. (2001). Apprentissage collaboratif à distance. Pour comprendre et concevoir les environnements d'apprentissage virtuels, Sainte-Foy, Québec, Presses Universitaires du Québec.

Jacquinot G. (1981). Présentation, Communications. (Apprendre des médias, Numéro thématique), 33, 1-4.

Jézégou, A. (2008). « Apprentissage autodirigé et formation à distance », Distances et savoirs, 63(3), p. 343-364.

Kadiyala M., Crynes B.L. (2000). “A review of literature on effectiveness of use of Information Technology in Education”, Journal of engineering education, 89(2), p. 177-190.

Kulik J., Kulik C., Cohen P. (1980). "Effectiveness of computer-based college teaching : A meta-analysis of findings", Review of Educational Research, 50, p. 525-544.

Lameul G. (2008). « Les effets de l'usage des technologies d'information et de communication en formation d'enseignants sur la construction des postures professionnelles », Savoirs, 17, p. 73-94.

Lebrun M. (2007). "Quality towards an expected harmony: Pedagogy and technology speaking together about innovation", $A A C E$ Journal, 15(2), p. 115-130. Chesapeake, VA: AACE. http://www.editlib.org/p/21024

Lebrun M. (2005). Théories et méthodes pédagogiques pour enseigner et apprendre: Quelle place pour les TIC dans l'éducation ? (2 édition revue), Bruxelles De Boeck.

Lebrun M., Docq F., Smidts D. (2009). "Claroline, an Internet Teaching and Learning Platform to Foster Teachers' Professional Development and Improve Teaching Quality: First Approaches”, AACE Journal, 17(4), p. 347-362. Chesapeake, VA: AACE. http://www.editlib.org/p/29355

Leech N., Dellinger A. B., Brannagan K.B. and Tanaka B. (2010). "Evaluating mixed research studies: a mixed methods approach", Journal of mixed methods research, 4(1) p. 17-31, Sage.

Merill D. (2000). Components of Instruction Toward a Theoretical Tool for Instructional Design, Department of Instructional Technology, Utah State University.

Meunier J.-P. et Peraya D. (1993). Introduction aux théories de la communication (2édition revue et augmentée, 2004). Bruxelles, De Boeck. 
Morgan G. (2003). Faculty use of course management systems, Research Study from the EDUCAUSE Center for Applied Research. http://connect.educause.edu/Library/ECAR/ FacultyUseofCourseManagem/39089

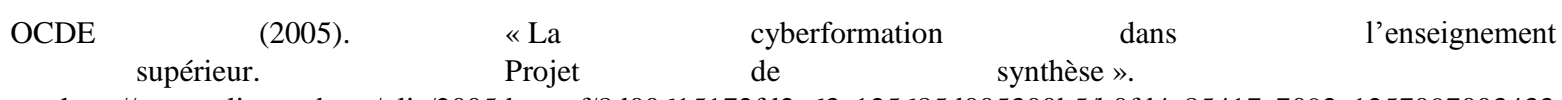
http://www.olis.oecd.org/olis/2005doc.nsf/8d00615172fd2a63c125685d005300b5/b0fd4e85417c7092c1257097003433c0/\$FIL E/JT00191859.PDF

Paquette G. (1993). «Les logiciels de formation ». G. Paquette (dir.), Initiation à la formation/conseil en milieu de travail (299315), Sainte-Foy, Télé-université.

Peraya D. (2010). «Des médias éducatifs aux environnements numériques de travail : Médiatisation et médiation ». V. Liquette (dir.), Médiation(s) et communication, Paris: Hermès.

Peraya D. (2000). «Le cyberespace : un dispositif de communication et de formation médiatisées », S. Alava (Ed.), Cyberespace et formations ouvertes. Vers une mutation des pratiques de formation, p. 17-44, Bruxelles, De Boeck.

Peraya D. et Campion, B. (2007a). «L'analyse des dispositifs hybrides : les effets d'un changement d'environnement virtuel de travail. D'un site Web à la plateforme Claroline », M. Frenay, B. Raucent et P. Wouters (dir.), Actes du $4^{e}$ colloque Questions de pédagogie dans l'enseignement supérieur. Les pédagogies actives : enjeux et conditions, Louvain-la-Neuve, Belgique, Presses universitaires de Louvain, p. 447- 456.

Peraya D. et Campion B. (2007b). «Perception d'un environnement virtuel par les étudiants de $2^{\mathrm{e}}$ cycle : quelle importance accordentils à l'usage d'un wiki ? ", Actes du $24^{e}$ Congrès de l'Association internationale de pédagogie universitaire [AIPU]. Vers un changement de culture en enseignement supérieur. Regards sur l'innovation, la collaboration et la valorisation, p. 2135, Montréal, Canada : Association internationale de pédagogie universitaire.

Pernin J. P. (2003). « Objets pédagogiques : unités d'apprentissage, activités ou ressources ? » Revue «Sciences et Techniques Educatives », Hors série 2003 « Ressources numériques, XML et éducation », p. 179-210.

Rabardel P. et Samurçay R. (2001). "From Artifact to Instrument-Mediated Learning", Symposium on New challenges to research on Learning, Helsinki.

Robertson A. (2006). Introduction aux banques d'objets d'apprentissage en français au Canada, Rapport péparé pour le compte du Réseau d'enseignement francophone à distance du Canada (REFAD).

Tardif J. (1996). «Une condition incontournable aux promesses des NTIC en apprentissage : une pédagogie rigoureuse », Actes de la Conférence d'ouverture au colloque de l'AQUOPS (Association québécoise des utilisateurs de l'ordinateur au primaire et au secondaire).

Wiley D. A. (2002). The Instructional use of learning Objects. http://www.reusability .org/read/ 